078

\section{WORKFLOW DIAGNOSTICO PER LA IDENTIFICAZIONE DI CRYPTOSPORIDIUM SPP. IN CAMPIONI FECALI}

Putignani L.'; Bordi E.'; Cacciò S.'; Paglia M.G.'; Boumis E.'; Petrosillo N.';Visca P. ${ }^{1,3}$

'Istututo Nazionale per lo studio delle Malattie Infettive, INMI,

"Lazzaro Spallanzani", Roma, Italia;

2Istituto Superiore di Sanità, ISS, Roma, Italia;

${ }^{3}$ Dipartimento di Biologia, Università "Roma Tre", Roma, Italia.

Introduzione. La criptosporidiosi rappresenta una frequente causa di morbidità e mortalità in individui immunocompromessi. Dall'introduzione della terapia antiretrovirale (HAART) tale parassitosi ha registrato una considerevole diminuzione di incidenza e un limitato impatto clinico sul paziente. In Italia, il rischio relativo di morte per criptosporidiosi è diminuito del $74 \%$ tra gli individui HIV+ nel periodo 1997-1998, quando la terapia HAART ha iniziato ad essere largamente usata. Nel nostro ospedale, l'Istituto Nazionale per lo Studio delle Malattie Infettive "Lazzaro Spallanzani" di Roma, abbiamo nell'ultimo anno registrato otto casi di criptosporidiosi in pazienti HIVpositivi. Tale incidenza ci ha indotto a sviluppare un protocollo diagnostico per l'identificazione di infezioni da Cryptosporidium spp. Metodi molecolari e microscopici sono stati combinati in un "workflow" operativo per l' $i$ dentificazione di specie di Cryptosporidium spp. in campioni fecali.

Lo scopo è stato:

i) produrre una diagnosi riproducibile migliorando la sensibilità dei metodi di "detection" microscopica già presenti e proponendo un nuovo protocollo identificativo di specie fino ad oggi assente;

ii) allargare la nostra conoscenza sul significato clinico ed epidemiologico di specie / genotipi/ sottotipi presenti nella comunità di HIV-positivi analizzata, attraverso un'analisi retrospettiva.

Metodi. I campioni fecali sono stati concentrati mediante acqua/etere, i preparati microscopici osservati sia a fresco che fissati. Per la genotipizzazione, sono stati messi a confronto diversi metodi di estrazione di DNA, per valutarne efficienza e resa, e sono stati usati come marcatori di "typing" i loci genici 18S rDNA, COWP e alcune sequenze microsatelliti.

Risultati. E' stato ottenuto un "morphotyping" su sporozoiti liberi e un utile metodo di genotipizzazione molecolare.

Conclusioni. Abbiamo sviluppato un "workflow" operativo per la "detection" e la identificazione di specie, genotipo, sottogruppo di Cryptosporidium spp. da campioni fecali conservati in formalina. Tale metodologia sviluppata fornisce nuovi strumenti per l'indagine di criptosporidiosi in parassitologia clinica.

\section{9}

\section{STRONGYLOIDES STERCORALIS E PROBLEMATICHE DIAGNOSTICHE: ESPERIENZA.}

\author{
Scalet G.', Pegoraro M. ${ }^{2}$, Fontana R. ' Stepan E. ${ }^{2}$ \\ 'Dip. di Patologia, Sez.di Microbiologia, \\ Facoltà di Medicina e Chirurgia, Università degli Studi di Verona. \\ ${ }^{2}$ Servizio di Microbiologia, \\ Ospedale Civile Maggiore, Az. Ospedaliera di Verona.
}

Introduzione. L'infestazione da Strongyloides stercoralis (Phylum Nematoda) può persistere in maniera asintomatica o paucisintomatica per anni; tuttavia in concomitanza ad un'alterazione delle condizioni immunitarie (anche di origine iatrogena) l'infestazione può evolvere in quadri di iperinfestazione: aumento del carico di larve, della loro disseminazione sistemica e, con essa, dei danni provocati dalla loro migrazione. La diagnosi, che consiste nel dimostrare la presenza di larve nelle feci, non può basarsi sulla concentrazione formolo-etere (FEA), perché essa ha una sensibilità in merito molto bassa. Al fine di ottimizzare la diagnostica di Strongyloides stercoralis abbiamo ritenuto opportuno, utilizzare metodiche specifiche di ricerca e questo Lavoro riporta i risultati relativi al periodo gennaio-maggio 2006.

Materiali. Sono stati selezionati 71 pazienti in base a: presenza di ipereosinofilia ( $>5 \%$ ); disturbi intestinali in soggetti di età superiore a 50 anni (per le abitudini di un tempo di camminare a piedi nudi o lavorare la terra con le mani nude); prurito alla pelle; richiesta specifica. Tali campioni sono stati sottoposti a: FEA, arricchimento secondo Baerman, coltura su agar.

Risultati. Abbiamo rilevato la presenza di larve di Strongyloides stercoralis in 2 pazienti (2.8\%). Di uno di questi è stato possibile seguire anche il follow-up terapeutico, utile per valutare la sensibilità dei diversi metodi impiegati. I primi 3 campioni di questo paziente risultavano positivi con tutte le metodiche applicate. Due settimane dopo il primo ciclo di terapia (Albendazolo $400 \mathrm{mg}$ bis in die per 2 giorni) la concentrazione secondo Baerman e la coltura si mantenevano positive, mentre FEA ha permesso di reperire solo rari esemplari larvali dopo osservazione di tutto il sedimento. Due settimane dopo il secondo ciclo di terapia l'arricchimento specifico e la coltura sono risultati ancora positivi in due campioni su tre, mentre FEA negativo in tutti. In seguito al terzo ciclo terapeutico i campioni della paziente risultavano negativi a tutti $\mathrm{i}$ metodi utilizzati.

Conclusioni. Riteniamo utile l'impiego di tecniche specifiche per la ricerca di Strongyloides stercoralis perchè più sensibili nel caso di bassa carica parassitaria o follow-up terapeutico. La coltura su agar ci sembra più agevole da eseguire rispetto all'arricchimento secondo Baerman nonostante necessiti dell'allestimento di almeno 5 piastre per campione ed un'attenta osservazione microscopica. 